\title{
A novel design of spacecraft combined attitude $\&$ sun tracking system using a versatile fuzzy controller
}

\begin{abstract}
Purpose

- The purpose of this paper is to develop a theoretical design for the alternative attitude control of the rotation about the pitch axis for the nadir-pointing spacecraft in the event of inertial actuator faults.

Design/methodology/approach

- This paper presents a novel and viable solution to that problem using the combined attitude and sun tracking system (CASTS) that was conceived from an engineering problemsolving toolkit called TRIZ. Linear and fuzzy controllers are used to test the spacecraft CASTS architecture. All the relevant governing equations of the control system and disturbance rejection methods are developed.
\end{abstract}

\section{Findings}

- The performance of the proposed CASTS control strategy is tested through numerical simulations. The results strongly suggest that the novel proposed control scheme is effective and promising for controlling the satellite attitude and sun tracking simultaneously in the presence of disturbance torques.

Research limitations/implications

- This work is mainly focused on the rigid body of the spacecraft hub that contains all attitude control hardware and payload instrumentation, and does not deal with the vibrations evolving from the propellant sloshing and large flexible appendages such as the deployable solar panels and synthetic aperture radar antennas.

Practical implications

- The results from this work reveal several practical applications worthy of reducing the weight, size of the spacecraft and, therefore, cost of missions while increasing the instrumentation capabilities.

Originality/value

- The proposed CASTS solution is a result of looking much wider than one system from a new combination of attitude control and sun tracking, as well as innovative ways of using it.

Keyword: Nonlinear control; Attitude control; Disturbance rejection methods; Fuzzy control; Satellite; Sun tracking 